Proceedings of the

International Geometry Center

Vol. 10, no. 1 (2017) pp. 59-66

\title{
Эквивалентность аффинной и грассмановой классификаций точек поверхности пространства Минковского
}

\author{
Стеганцева П. Г., Гречнева М. А.
}

\begin{abstract}
The article deals with the problem, connected with the classification of the points of the two-dimensional surfaces in the fourdimensional Minkowski space. The affine classification and classification with the help of the Glassman image have been made. The conditions of the equivalence of these classifications have been found.
\end{abstract}

\begin{abstract}
Аннотация. В данной работе рассматривается задача классификации точек двумерных поверхностей четырехмерного пространства Минковского. Получены аффинная классификация и классификация с помощью грассманова образа поверхности. Найдены условия, при которых эти две классификации эквивалентны.
\end{abstract}

Вопрос о классификации точек многообразия является одним из основных в дифференциальной геометрии погруженных многообразий. По типу точки на многообразии можно сделать вывод о виде окрестности этой точки на многообразии. В дифференциальной геометрии гиперповерхностей этот вопрос решается несколькими способами: по числу асимптотических направлений в ней, по знаку и значениям главных кривизн гиперповерхности, с помощью гауссовой кривизны, по виду соприкасающегося параболоида. Важно отметить, что в этом случае все эти способы дают один и тот же результат.

Иначе обстоит дело, когда размерность поверхности меньше размерности пространства более чем на единицу. В этом случае не всегда можно разбить точки поверхности на конечное число классов. Те случаи, когда такое разбиение возможно, описаны в работе [2]. В работе [1] рассмотрена классификация точек грассманова образа поверхности. В

Ключевые слова: пространство Минковского, грассманов образ 
работе [3] приведена аффинная классификация точек двумерных поверхностей четырехмерного евклидова пространства по виду соприкасающегося параболоида, а также указывается на эквивалентность этой классификации и классификации с помощью грассманова образа поверхности.

В данной работе рассмотрим вопрос о классификации точек двумерных поверхностей, заданных в пространстве Минковского.

\section{1. АФФИННАЯ И ГРАССМАНОВА КЛАССИФИКАЦИИ ТОЧЕК ПОВЕРХНОСТИ ПРОСТРАНСТВА МИНКОВСКОГО}

Аффинное четырехмерное пространство со скалярным произведением вида $(\bar{x}, \bar{y})=-x_{1} y_{1}+x_{2} y_{2}+x_{3} y_{3}+x_{4} y_{4}$ называется пространством Минковского и обозначается ${ }^{1} R_{4}$. В этом пространстве все векторы делятся на три класса: пространственноподобные $\left(\bar{x}^{2}>0\right)$, времениподобные $\left(\bar{x}^{2}<0\right)$ и изотропные $\left(\bar{x}^{2}=0\right)$. Тип двумерной плоскости определяется парой ортогональных векторов $\bar{x}, \bar{y}$. Плоскость называется пространственноподобной (времениподобной, или изотропной), если $\bar{x}^{2}>0, \bar{y}^{2}>0\left(\bar{x}^{2}<0, \bar{y}^{2}>0\right.$, или $\left.\bar{x}^{2}=0, \bar{y}^{2}>0\right)$. Тип двумерной поверхности будем определять по типу касательной плоскости. Двумерная поверхность пространства ${ }^{1} R_{4}$ называется пространственноподобной (времениподобной или изотропной), если касательная плоскость к ней в каждой точке является пространственноподобной (времениподобной или изотропной).

Пусть $V^{2}$ - неизотропная поверхность класса $C^{k}, k \geq 1$ в ${ }^{1} R_{4}$, заданная векторным уравнением $\bar{r}=\bar{r}\left(u^{1}, u^{2}\right)$. Векторы $\bar{r}_{i}=\frac{\partial \bar{r}}{\partial u^{2}}$ являются касательными к поверхности. Рассмотрим в каждой точке $x \in V^{2}$ нормальную плоскость $N_{x}$ и выберем в ней линейно независимые векторы $\bar{\xi}_{1}$ и $\bar{\xi}_{2}$ так, чтобы четверка векторов $\bar{r}_{1}, \bar{r}_{2}, \bar{\xi}_{1}, \bar{\xi}_{2}$ была ортонормированной в ${ }^{1} R_{4}$. Если поверхность пространственноподобная, то нормальные плоскости в каждой точке этой поверхности будут времениподобными, если же поверхность времениподобна, то нормальные плоскости пространственноподобные.

С помощью каждого вектора нормали определим вторую квадратичную форму

$$
I I^{k}=\left(\overline{r_{i j}}, \bar{\xi}_{k}\right) d u^{i} d u^{j}, i, j, k=1,2 .
$$

Матрицы этих форм имеют вид $A^{k}=\left(\begin{array}{cc}L_{11}^{k} & L_{12}^{k} \\ L_{21}^{k} & L_{22}^{k}\end{array}\right)$, где $L_{i j}^{k}=\left(\bar{r}_{i j}, \bar{\xi}_{k}\right)$.

Две точки регулярной поверхности называются аффинно эквивалентными, если соприкасающиеся параболоиды в этих точках можно отобразить друг на друга невырожденным аффинным преобразованием в объемлющем пространстве [2]. 
Пусть $Q$ - точка времениподобной поверхности $V^{2} \subset{ }^{1} R_{4}$. Выберем систему координат в пространстве ${ }^{1} R_{4}$ так, чтобы точка $Q$ совпала с началом координат, а касательное пространство $T_{Q} V^{2}-$ с подпространством $\left\{x^{1}, x^{2}\right\}$. Тогда уравнения соприкасающегося параболоида с вершиной в точке $Q$ будут иметь вид

$$
x^{k+2}=L_{i j}^{k} x^{i} x^{j} .
$$

Рассмотрим пучок вторых квадратичных форм $A^{1}-\lambda A^{2}$ поверхности $V^{2} \subset{ }^{1} R_{4}$. Тогда в каждой точке $x \in V^{2}$ уравнение соприкасающегося параболоида невырожденным аффинным преобразованием объемлющего пространства приводится к одному из следующих канонических видов (в зависимости от вида элементарных делителей пучка):

1) $x^{3}=\left(x^{1}\right)^{2}, x^{4}=\left(x^{2}\right)^{2}$, для случая элементарных делителей $\lambda-\lambda_{1}$, $\lambda-\lambda_{2}, \lambda_{1} \neq \lambda_{2}, \lambda_{1}, \lambda_{2} \in R$

2) $x^{3}=2 x^{1} x^{2}, x^{4}=\left(x^{2}\right)^{2}$, если имеем один линейный элементарный делитель кратности 2 , то есть $\left(\lambda-\lambda_{1}\right)^{2}, \lambda_{1} \in R$;

3) $x^{3}=2 x^{1} x^{2}, x^{4}=\left(x^{1}\right)^{2}-\left(x^{2}\right)^{2}$, для квадратичного элементарного делителя $\lambda^{2}-2 \alpha \lambda+\left(\alpha^{2}+\beta^{2}\right), \beta \neq 0$.

В случае пространственноподобной поверхности систему координат в пространстве ${ }^{1} R_{4}$ будем выбирать так, чтобы касательное пространство $T_{Q} V^{2}$ совпадало с подпространством $\left\{x^{3}, x^{4}\right\}$. Тогда уравнения соприкасающегося параболоида с вершиной в точке $Q$ будут иметь вид

$$
x^{k}=L_{i j}^{k} x^{i+2} x^{j+2} .
$$

В каждой точке $x \in V^{2}$ уравнение соприкасающегося параболоида невырожденным аффинным преобразованием объемлющего пространства приводится к одному из следующих канонических видов:

1) $x^{1}=\left(x^{3}\right)^{2}, x^{2}=\left(x^{4}\right)^{2}$, для случая элементарных делителей $\lambda-\lambda_{1}$, $\lambda-\lambda_{2}, \lambda_{1} \neq \lambda_{2}, \lambda_{1}, \lambda_{2} \in R$

2) $x^{1}=2 x^{3} x^{4}, x^{2}=\left(x^{4}\right)^{2}$, если имеем один линейный элементарный делитель кратности 2 , то есть $\left(\lambda-\lambda_{1}\right)^{2}, \lambda_{1} \in R$;

3) $x^{1}=2 x^{3} x^{4}, x^{2}=\left(x^{3}\right)^{2}-\left(x^{4}\right)^{2}$, для квадратичного элементарного делителя $\lambda^{2}-2 \alpha \lambda+\left(\alpha^{2}+\beta^{2}\right), \beta \neq 0$.

Таким образом, точки поверхности можно разбить на три класса. Такая классификация точек называется афбинной. Заметим, что этот результат ничем не отличается от случая евклидова пространства [3], так как евклидово пространство и пространство Минковского имеют одни и те же аффинные свойства.

Далее рассмотрим еще одну классификацию точек поверхности, которую будем называть грассмановой. Этот термин объясняется тем, что тип точки поверхности определяется типом точек грассманова образа 
этой поверхности. Поставим в соответствие каждой точке поверхности $V^{2}$ пространства ${ }^{1} R_{4}$ плоскость, параллельную нормальной плоскости $N_{x}$ и проходящую через фиксированную точку пространства ${ }^{1} R_{4}$. Этим устанавливается отображение поверхности $V^{2}$ в грассманово многообразие $P G(2,4)$ двумерных плоскостей пространства ${ }^{1} R_{4}$, подмногообразиями которого будут многообразия времениподобных плоскостей — ${ }^{T} P G(2,4)$, пространственноподобных плоскостей — ${ }^{S} P G(2,4)$ и изотропных плоскостей — Is $P G(2,4)$. Грассмановым образом поверхности $V^{2}$ называют образ этого отображения. Будем считать, что якобиан указанного отображения отличен от нуля. Таким образом, грассманов образ является регулярной двумерной поверхностью $\Gamma^{2}$, которая зависит от тех же параметров $u^{1}, u^{2}$, что и поверхность $V^{2}$. Изучению грассманова образа неизотропных поверхностей пространства Минковского посвящена работа [6].

Предположим, что существуют области на $V^{2}$, точечный грассманов образ которых имеет во всех точках касательные плоскости одного типа. Заметим, что тип точек грассманова образа может как совпадать с типом поверхности $V^{2}$, так и отличаться от него.

Определение 1.1. Точка $x$ поверхности $V^{2} \subset{ }^{1} R_{4}$ называется эллиптической (параболической, гиперболической), если точка грассманова образа поверхности, соответствующая этой точке, является эллиптической (параболической, гиперболической).

В работе [6] были сформулированы

Определение 1.2. Точка грассманова образа $\Gamma^{2}$ времениподобной поверхности $V^{2}$ называется эллиптической (параболической, гиперболической), если для площадки, касательной к $\Gamma^{2}$ в этой точке, секционная кривизна грассманова подмногообразия ${ }^{S} P G(2,4)$ удовлетворяет условию $K(\sigma)<1(K(\sigma)=1, K(\sigma)>1)$.

Определение 1.3. Точка грассманова образа $\Gamma^{2}$ пространственноподобной поверхности $V^{2} \subset{ }^{1} R_{4}$ называется эллиптической (параболической, гиперболической), если для площадки, касательной к $\Gamma^{2}$ в этой точке, секционная кривизна грассманова подмногообразия ${ }^{T} P G(2,4)$ удовлетворяет условию $K(\sigma)>-1(K(\sigma)=-1, K(\sigma)<-1)$.

\section{2. ДОКАЗАТЕЛЬСТВО ЭКВИВАЛЕНТНОСТИ АФФИННОЙ И ГРАССМАНОВОЙ КЛАССИФИКАЦИЙ ТОЧЕК ПОВЕРХНОСТИ}

Рассмотрим условия, при которых первому (второму, третьему) классу точек в аффинной классификации соответствуют эллиптические (параболические, гиперболические) точки в грассмановой классификации. 
В этом случае будем говорить об эквивалентности аффинной и грассмановой классификаций точек.

Теорема 2.1. Для времениподобной поверхности с пространственноподобным грассмановым образом афбинная и грассманова классификаици эквивалентны.

Доказательство. Аффинная классификация дает три класса точек поверхности $V^{2} \subset{ }^{1} R_{4}$, каждый из которых определяется видом соприкасающегося параболоида. Поскольку соприкасающийся параболоид с вершиной в точке поверхности и сама поверхность имеют в этой точке общую касательную плоскость, а значит и общую нормальную плоскость, то для определения типа точки $x$ поверхности в грассмановой классификации необходимо найти значение секционной кривизны грассманова многообразия вдоль площадки, касательной к грассмановому образу соприкасающегося параболоида в точке, соответствующей точке $x \in V^{2}$.

Отметим, что не всегда есть возможность пользоваться каноническими уравнениями соприкасающегося параболоида. Это объясняется тем, что система координат, относительно которой уравнение параболоида имеет канонический вид, может индуцировать такую систему координат на грассмановом многообразии, относительно которой касательная плоскость к грассманову образу имеет вырожденную метрику.

Рассмотрим времениподобную поверхность $V^{2} \subset{ }^{1} R_{4}$ с пучком вторых квадратичных форм $A^{1}-\lambda A^{2}$. В случае различных линейных элементарных делителей $\lambda-\lambda_{1}, \lambda-\lambda_{2}$ этого пучка матрицы $A^{1}, A^{2}$ можно привести к виду [5]:

$$
A^{1}=\left(\begin{array}{cc}
l_{1} \lambda_{1} & 0 \\
0 & l_{2} \lambda_{2}
\end{array}\right), \quad A^{2}=\left(\begin{array}{cc}
l_{1} & 0 \\
0 & l_{2}
\end{array}\right), \quad l_{i}= \pm 1
$$

Тогда векторное уравнение соприкасающегося параболоида принимает вид

$$
\bar{r}\left(u^{1}, u^{2}\right)=\left(u^{1}, u^{2}, l_{1} \lambda_{1}\left(u^{1}\right)^{2}+l_{2} \lambda_{2}\left(u^{2}\right)^{2}, l_{1}\left(u^{1}\right)^{2}+l_{2}\left(u^{2}\right)^{2}\right) .
$$

Для вычисления секционной кривизны грассманова многообразия вдоль площадки, касательной к грассманову образу параболоида, воспользуемся формулой [4]

$$
K(\sigma)=\frac{\left(-\sigma^{12}+\sigma^{34}\right)^{2}-\left(\sigma^{13}+\sigma^{24}\right)^{2}}{\left(\sigma^{12}\right)^{2}-\left(\sigma^{13}\right)^{2}-\left(\sigma^{14}\right)^{2}-\left(\sigma^{23}\right)^{2}-\left(\sigma^{24}\right)^{2}+\left(\sigma^{34}\right)^{2}}
$$


где $\sigma^{a b}=x^{[a} y^{b]}$ - координаты бивектора $\bar{\sigma}$, определяемого направляющими векторами $\bar{X}=\left(x^{a}\right)$ и $\bar{Y}=\left(y^{a}\right), a=1,2,3,4$ касательной площадки. Координаты $\sigma^{a b}$ удовлетворяют условию Плюккера

$$
\sigma^{12} \sigma^{34}-\sigma^{13} \sigma^{24}+\sigma^{14} \sigma^{23}=0 .
$$

Грассманово многообразие $P G(2,4)$ представляет собой алгебраическую поверхность с метрикой сигнатуры $(--++),[4]$. Бивектор $\bar{\sigma}$ можно рассматривать как точку шестимерного пространства. Псевдориманова метрика грассманова многообразия индуцирует в этом шестимерном пространстве метрику сигнатуры $(+----+)$. Тогда выражение, стоящее в знаменателе последней дроби, представляет собой скалярный квадрат бивектора $\bar{\sigma}$, который и определяет касательную площадку к грассманову образу.

В случае евклидова пространства показано, что координаты касательных векторов $\bar{X}$ и $\bar{Y}$ представляются через коэффициенты первой и вторых квадратичных форм [1]. В нашем случае эти представления имеют вид

$$
\begin{aligned}
& \bar{X}=\left(\frac{L_{11}^{2}}{\sqrt{g_{11}}},-\frac{L_{11}^{1}}{\sqrt{g_{11}}}, \frac{L_{12}^{2}}{\sqrt{g_{22}}},-\frac{L_{12}^{1}}{\sqrt{g_{22}}}\right), \\
& \bar{Y}=\left(\frac{L_{12}^{2}}{\sqrt{g_{11}}},-\frac{L_{12}^{1}}{\sqrt{g_{11}}}, \frac{L_{22}^{2}}{\sqrt{g_{22}}},-\frac{L_{22}^{1}}{\sqrt{g_{22}}}\right) .
\end{aligned}
$$

То есть касательные векторы к грассманову образу рассматриваемого параболоида имеют вид

$$
\bar{X}=\left(\frac{2 l_{1}}{\sqrt{g_{11}}},-\frac{2 l_{1} \lambda_{1}}{\sqrt{g_{11}}}, 0,0\right), \quad \bar{Y}=\left(0,0, \frac{2 l_{2}}{\sqrt{g_{22}}},-\frac{2 l_{2} \lambda_{2}}{\sqrt{g_{22}}}\right) .
$$

Тогда бивектор $\bar{\sigma}$ имеет координаты:

$$
\begin{aligned}
& \sigma^{12}=0, \\
& \sigma^{13}=\frac{4 l_{1} l_{2}}{\sqrt{g_{11} g_{22}}}, \quad \sigma^{14}=-\frac{4 l_{1} l_{2} \lambda_{2}}{\sqrt{g_{11} g_{22}}}, \\
& \sigma^{23}=-\frac{4 l_{1} l_{2} \lambda_{1}}{\sqrt{g_{11} g_{22}}}, \quad \sigma^{24}=\frac{4 l_{1} l_{2} \lambda_{1} \lambda_{2}}{\sqrt{g_{11} g_{22}}}, \quad \sigma^{34}=0 .
\end{aligned}
$$

Секционная кривизна вдоль площадки, определяемой векторами $\bar{X}$ и $\bar{Y}$, в соответствии с формулой $(2.1)$, будет равна

$$
K(\sigma)=\frac{\left(1+\lambda_{1} \lambda_{2}\right)^{2}}{\left(1+\lambda_{1}^{2}\right)\left(1+\lambda_{2}^{2}\right)} .
$$

Очевидно, что $K(\sigma)<1$ при любых $\lambda_{1} \neq \lambda_{2}$. Таким образом, первому классу точек в аффинной классификации соответствуют эллиптические точки в грассмановой классификации. 
Пусть теперь секционная кривизна грассманова многообразия вдоль площадки, касательной к грассманову образу поверхности, удовлетворяет неравенству $K(\sigma)<1$, или

$$
\frac{\left(-\sigma^{12}+\sigma^{34}\right)^{2}-\left(\sigma^{13}+\sigma^{24}\right)^{2}}{\left(\sigma^{12}\right)^{2}-\left(\sigma^{13}\right)^{2}-\left(\sigma^{14}\right)^{2}-\left(\sigma^{23}\right)^{2}-\left(\sigma^{24}\right)^{2}+\left(\sigma^{34}\right)^{2}}<1 .
$$

По условию теоремы грассманов образ пространственноподобен, а значит и касательная площадка $\sigma$ тоже пространственноподобна, то есть выражение в знаменателе положительно.

Поэтому неравенство (2.3), с учетом условия Плюккера, можно привести к виду

$$
\left(\sigma^{14}+\sigma^{23}\right)^{2}<4 \sigma^{13} \sigma^{24} .
$$

Если записать координаты бивектора $\bar{\sigma}$ по представлениям (2.2) векторов $\bar{X}$ и $\bar{Y}$, то последнее неравенство примет вид

$$
\left(2 L_{12}^{1} L_{12}^{2}-L_{11}^{2} L_{22}^{1}-L_{11}^{1} L_{22}^{2}\right)^{2}>4\left(L_{11}^{1} L_{22}^{1}-\left(L_{12}^{1}\right)^{2}\right)\left(L_{11}^{2} L_{22}^{2}-\left(L_{12}^{2}\right)^{2}\right) \text {. }
$$

Запишем детерминант пучка вторых квадратичных форм

$$
\begin{aligned}
\left|A^{1}-\lambda A^{2}\right|= & \left|\begin{array}{ll}
L_{11}^{1}-\lambda L_{11}^{2} & L_{12}^{1}-\lambda L_{12}^{2} \\
L_{12}^{1}-\lambda L_{12}^{2} & L_{22}^{1}-\lambda L_{22}^{2}
\end{array}\right|= \\
= & \lambda^{2}\left(L_{11}^{2} L_{22}^{2}-\left(L_{12}^{2}\right)^{2}\right)+\lambda\left(2 L_{12}^{1} L_{12}^{2}-L_{11}^{2} L_{22}^{1}-L_{11}^{1} L_{22}^{2}\right)+ \\
& +\left(L_{11}^{1} L_{22}^{1}-\left(L_{12}^{1}\right)^{2}\right) .
\end{aligned}
$$

Он представляет собой квадратный трехчлен относительно $\lambda$. Тогда условие (2.4) означает, что дискриминант этого квадратного трехчлена больше нуля, то есть пучок вторых квадратичных форм имеет два различных линейных элементарных делителя.

Рассмотрим второй класс точек аффинной классификации. Ему будет соответствовать класс параболических точек в грассмановой классификации. Действительно, уравнение соприкасающегося параболоида в этом случае имеет вид

$$
\bar{r}\left(u^{1}, u^{2}\right)=\left(u^{1}, u^{2}, 2 l_{1} \lambda_{1} u^{1} u^{2}+l_{1}\left(u^{2}\right)^{2}, 2 l_{1} u^{1} u^{2}\right) .
$$

Секционная кривизна вдоль площадки, касательной к грассманову образу этой поверхности, равна 1. Это равенство достигается тогда и только тогда, когда дискриминант детерминанта пучка вторых квадратичных форм равен нулю.

Точки третьего класса в аффинной классификации соответствуют гиперболическим точкам в грассмановой классификации. Действительно, используя соответствующее уравнение соприкасающегося параболоида, можно найти значение секционной кривизны вдоль площадки, 
касательной к грассманову образу этого параболоида, в виде

Очевидно, что $K(\sigma)>1$.

$$
K(\sigma)=1+\frac{4 \beta^{2}}{\left(1+\alpha^{2}+\beta^{2}\right)^{2}} .
$$

В обратную сторону, из того, что точка гиперболическая, следует, что дискриминант детерминанта пучка вторых квадратичных форм меньше нуля, то есть пучок имеет квадратичный элементарный делитель.

Теорема 2.2. Для пространственноподобной поверхности с времениподобным грассмановым образом афбинная и грассманова классификаиии эквивалентны.

Доказательство аналогично доказательству теоремы 2.1.

\section{ЛитерАТУРА}

[1] Ю. А. Аминов. Геометрия подмногообразий. Наукова думка, 2002.

[2] А. А. Борисенко. Афинная классификация точек многомерных поверхностей. Сиб. мат. журн., 342(3):76-82, 1974.

[3] А. А. Борисенко, Ю. А. Николаевский. Классификация точек трехмерных поверхностей по грассманову образу. Укр. геом. сборник, 32:11-27, 1989.

[4] И. Маазикас. K римановой геометрии грассмановых многообразий неизотропных подпространств псевдоевклидова пространства. Ученые записки Тартусского университета, (342):76-82, 1974.

[5] А. З. Петров. Пространства Эйнштейна. Физматгиз, 1961.

[6] П. Г. Стеганцева, М. А. Гречнева. Грассманов образ неизотропной поверхности псевдоевклидова пространства. Известия высших учебных заведений. Математика, (2):65-75, 2017.

Поступила в редакиию 15 февраля 2017, принята к печати 18 марта 2017.

Стеганцева Полина Георгиевна

ЗАПОРОЖСКИЙ НАЦИОНАЛЬНЫЙ УНИВЕРСИТЕТ, Г. ЗАПОРОЖЬЕ, УЛ. ЖУКОВСКОГО, 66, УКРАИНА

Email: steg_pol@mail.ru

Гречнева Марина Александровна

ЗАПОРОЖСКИЙ НАЦИОНАЛЬНЫЙ УНИВЕРСИТЕТ, Г. ЗАПОРОЖЬЕ, УЛ. ЖУУОВСКОГО, 66, УКРАИНА

Email: mag83@list.ru 